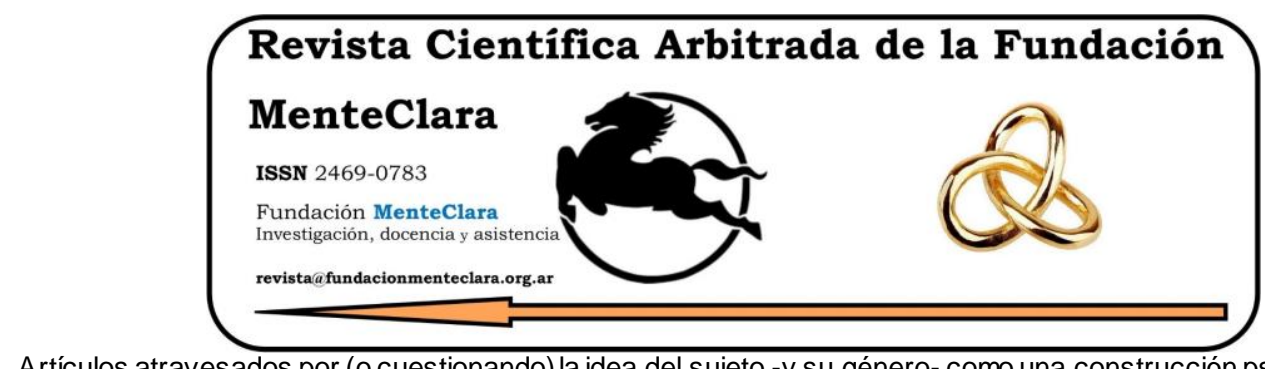

Artículos atravesados por (o cuestionando) la idea del sujeto -y su género- como una construcción psicobiológica de la cultura. Articles driven by (or questioning) the idea of the subject -and their gender- as a cultural psychobiological construction.

Vol. 6 (2021), enero-diciembre ISSN 2469-0783

https: / / datahub.io/ dataset/2021-6-e239

\title{
APOYO SOCIAL Y ENVEJECIMIENTO ACTIVO: APROXIMACIONES DESDE LA PERSPECTIVA DE LA PSICOLOGÍA POSITIVA
}

\author{
SOCIAL SUPPORT AND ACTIVE AGING: APPROXIMATIONS FROM POSITIVE \\ PSYCHOLOGY PERSPECTIVE
}

Andrés Guido González Zabala andres.gzabala@gmail.com

Estudiante de Psicología, Cátedra Psicología de la Salud, Universidad de Flores, Argentina.

Cómo citar este artículo / Citation: González Zabala, A. (2021). Apoyo social y envejecimiento activo: Aproximaciones desde la perspectiva de la psicología positiva. Revista Cientifica Arbitrada de la Fundación MenteClara, Vol. 6 (239). DOI:

https: / / doi.org/ 10.32351/rca.v6.239

Copyright: (C) 2021 RCAFMC. Este artículo de acceso abierto es distribuido bajo los términos de la licencia Creative Commons Attribution 4.0 International License (CC BY 4.0). Recibido: 10/07/2021. Aceptado: 18/07/2021 Publicación online: 21/07/2021

Conflicto de intereses: Ninguno que declarar.

\section{Resumen}

El presente trabajo intenta dar un panorama general, desde la óptica de la psicología positiva, sobre los conceptos de envejecimiento activo y apoyo social. A su vez, mediante la revisión de diversos artículos de investigación se intentará relacionar con claridad y fundamentos ambas construcciones teóricas. Los resultados hallados convergen en un punto común, hay una fuerte interrelación entre ellas. Para que el envejecimiento activo sea posible, es necesario que exista apoyo social en mayor o menor medida. A raíz de lo dicho, surge la necesidad de tomar conciencia sobre la importancia que tienen las redes de contención en la sociedad general y puntualmente en los adultos mayores. 


\begin{abstract}
The present work tries to provide a glimpse, through positive psychology, about the concepts of active aging and social support. At the same time, using a variety of research articles we will try to link both with clarity and fundamentals those theoretical constructions. The results found converge on a common point, there is a strong interrelation between them. For active aging to be possible, social support is necessary to exist to a greater or lesser extent. As a result of what has been said, there is a need to become aware of the importance of containment nets in general society and specifically in older adults.
\end{abstract}

Palabras Claves: Envejecimiento Activo; Apoyo Social

Keyw ords: Active Aging; Social Support 


\section{Introducción}

Este artículo de revisión bibliográfica surge con la motivación de aportar y colaborar al campo de la psicología positiva. La meta es sistematizar y promover los hallazgos hasta ahora encontrados sobre el apoyo social y el envejecimiento activo en el campo de la salud mental. Abordaremos ambos constructos teóricos tratando de dar luz a sus significados e interrelaciones. Analizaremos investigaciones realizadas en diferentes partes del mundo que nos permitirán seguir formando y reformulando estos conceptos. Por último, resaltaremos algunos de los que creemos los principales puntos en común entre ambos constructos según la perspectiva de diversos autores.

\section{Envejecimiento Activo}

Para abordar el concepto de Envejecimiento Activo, debemos remontarnos a su origen. ¿Cómo surge este constructo? Surge alineado al paradigma positivo del envejecimiento, de la mano de diferentes estudios científicos sobre las posibles maneras de desarrollarse de manera óptima como seres humanos. El proceso de elaboración teórica tuvo como ideas circundantes al envejecimiento 'productivo' y al envejecimiento 'saludable'. Pero finalmente prevaleció una tendencia a utilizar el término 'activo'. Con él se intenta ligar dos aspectos claves: el hecho mismo de realizar actividad y la potencialidad de ser protagonistas de la propia vida y el proceso de envejecimiento. El fin fundamental del concepto es defender la posibilidad de mantenerse activo y sano en la edad adulta. Para que esto ocurra es necesario que se creen y mantengan oportunidades reales y concretas dentro de la sociedad. Oportunidades que permitan a las personas mayores estar activas y en movimiento. Se trata de que puedan hacerse cargo de sus propias vidas, hasta que su 
edad y capacidades se lo permitan (Marsillas et al., 2017). El principio troncal del envejecimiento activo es una mejor salud y vida social para los adultos mayores (Lin \& Huang, 2016). Al lograr que participen activamente en la sociedad se revalorizan y utilizan sus conocimientos. Es importante potenciar, y evitar que pasen desapercibidas, aquellas competencias que poseen los adultos mayores para ser partícipes en la sociedad de manera activa y mejorar su propia calidad de vida (del Barrio et al., 2018).

La Organización Mundial de la Salud en 2002 formuló la siguiente definición: "El envejecimiento activo es el proceso de optimizar las oportunidades de salud, participación y seguridad para mejora la calidad de vida a medida que las personas envejecen" (World Health Organisation, 2002). Este documento también hace mención de las políticas y programas vinculados: "las políticas y programas deben basarse en los derechos, necesidades, preferencias y capacidades de las personas mayores". Desde la perspectiva de la OMS, se apunta a ir más allá de la productividad y de la salud en la edad adulta. El horizonte está puesto en una participación continua y activa en todas las dimensiones de la sociedad: lo social, lo económico, lo cultural, lo espiritual y lo cívico.

Cabe destacar, como lo hacen Rantanen et al. (2019), que toda definición de envejecimiento activo a la que se arribe debe contemplar los objetivos de la persona, las capacidades funcionales que posee, el grado de autonomía que maneja y las actividades que realiza en la actualidad.

Por otra parte, según lo indican estudios realizados, en la actualidad es cada vez más visible la exigencia por parte de las nuevas generaciones 
de personas mayores de una mejor calidad de vida. Reclaman espacios en los que puedan aportar positivamente a la sociedad. Las demandas de las generaciones de adultos mayores suelen converger en un punto común: poder desarrollarse y contribuir a la sociedad siendo ellos mismos, desempeñando un rol en el que sean reconocidos (del Barrio et al., 2018).

En numerosos países, sobre todo en Europa, incrementa de manera continua la defensa del concepto. Se lo considera un medio para concientizar respecto a la importancia de mantener y optimizar las oportunidades de las personas que envejecen (Lin \& Huang, 2016). Sin embargo, para que el concepto no quede incompleto, debe contemplar múltiples factores como, por ejemplo, la autosuficiencia, el compromiso con la sociedad, la espiritualidad, la seguridad financiera, un estilo de vida saludable y el fortalecimiento de los lazos familiares, entre otros Rantanen et al. (2019). Si no se contemplara la seguridad financiera, por ejemplo, tal como remarcan del Barrio et al. (2018), podría ocurrir que el discurso del Envejecimiento Activo se tornara excluyente para un amplio grupo de la sociedad. No perdamos de vista que el estilo de vida que se promueve muchas veces es posible y alcanzable sólo para determinadas personas, aquellas que cuenten con los medios económicos suficientes y las capacidades fisicas y mentales necesarias.

Para evitar, o en su defecto disminuir la discriminación y exclusión que pueda aparecer, es necesaria la puesta en marcha de dispositivos institucionales. Estos deben facilitar, y en algunos casos hacer posible, la participación de personas mayores en su entorno y comunidad. En consecuencia, podemos afirmar que es indispensable contar con políticas 
e intervenciones por parte de los gobiernos locales (del Barrio et al., 2018). Al conocer mejor las necesidades de las personas adultas pueden tomarse decisiones más eficaces respecto a los recursos disponibles. Por ejemplo, según lo indica la investigación realizada por Tkatch et al. (2017), en cuestiones de costos de atención médica, aquellos adultos mayores que informan altos niveles de satisfacción con la vida, junto con redes de apoyo social sólidas y niveles de estrés bajos, tienen más posibilidades de utilizar los servicios de atención médica preventiva. Esto conlleva a detecciones tempranas y prevención de enfermedades que, por consecuencia, resultarán en un menor costo de atención médica. Por otra parte, en aquellos adultos mayores en los que hay altos niveles de depresión y bajos niveles de apoyo social, se puede ver mayor utilización de atención médica curativa, en vez de preventiva. Y como resultado, mayores costos debido a la necesidad de tratamientos más complejos y prolongados.

En conclusión, podemos afirmar que el envejecimiento activo promueve el bienestar, o en su defecto, lo mantiene (Rantanen et al., 2019). Gozar de plena ciudadaníay participar activamente en la sociedad es un derecho por parte de las personas mayores. Su presencia en la dinámica social y la política de su comunidad logra visibilizarlos como sujetos sociales y dejar atrás el lugar de mero objetos de atención y administración (del Barrio et al., 2018).

\section{Apoyo social}

Se pueden encontrar una gran variedad definiciones respecto al apoyo social, lo que denota la complejidad de su conceptualización. En principio, podemos afirmar que es un recurso del tipo psicológico y social 
vinculado a las relaciones interpersonales y a las redes sociales de las personas (Kocalevent et al., 2018). La definición formulada por Kang et al. (2018), lo define como la percepción de estar recibiendo asistencia afectiva y actitudinal por determinado sujeto receptor. Dicha asistencia implica empatía, cariño, amor, ayudas tangibles y compañía. García Torres et al. (2017) sostienen el apoyo social requiere de la existencia o la disponibilidad de personas en las que se pueda confiar. Personas mediante las cuales el sujeto pueda sentirse respaldado y amado, y tenga la tranquilidad de saber que se preocupan por él.

En el constructo teórico de apoyo social hay dos componentes que no pueden dejar de tenerse en cuenta: apoyo estructural y apoyo funcional. Este último, el funcional, está relacionado con cuál es la experiencia percibida del sujeto por parte de una determinada red social. De qué manera esta red acciona e influye en su calidad de vida. Qué tanto y cómo se satisface la expectativa de obtener apoyo por parte de la familia, de amigos y/o de la comunidad cuando se lo necesita (Kocalevent et al., 2018). Los elementos claves del apoyo funcional son: el aspecto emocional, el informativo y el compañerismo (Milgrom et al., 2019). Por otra parte, el apoyo estructural, se refiere más precisamente al tamaño y al tipo de red social en la que se busca el sostén, como así también con qué frecuencia se entra en contacto. Sus componentes tienen que ver con la existencia de las relaciones en sí mismas y sus características en términos, sobre todo, cuantitativos (Kocalevent et al., 2018).

Como sostienen Tkatch et al. (2017), las redes sociales de cada individuo son las que proporcionan este recurso tan valioso. Recurso capaz de brindar sensación de seguridad y que permite la confianza 
suficiente para creer que en aquellos momentos de necesidad habrá alguien disponible para brindar su atención y ayuda.

La ausencia de apoyo social puede tener consecuencias en el bienestar físico y mental de las personas. Con la edad se hace más posible la pérdida de salud, parejas, amigos, e incluso, la independencia. Estas pérdidas pueden colocar a los adultos mayores en riesgo de soledad y aislamiento, e influir de manera significativamente negativa en sus vidas (Smith, 2012). Según Czaja et al. (2018) es necesario tener en cuenta que tanto el aislamiento social, como la soledad, son problemas que enfrentan muchos adultos mayores en la actualidad.

En las investigaciones de Alsubaie et al. (2019) se sostiene que el apoyo social actúa de manera positiva frente a situaciones estresantes de la vida y que promueve la salud mental. Cumple un rol esencial en la calidad de vida, ya que colabora con la sensación de sentirse apreciado y conectado al entorno. Tiene un alto impacto en personas con depresión y se puede concluir que tanto su presencia como su ausencia son determinantes en cuestiones de salud mental.

Queda entonces claro que el apoyo social juega un papel protector contra la depresión en la población general (Milgrom et al., 2019).

Hay una distinción del tipo cultural que puede alentar o desalentar que prevalezca el apoyo social dentro de una sociedad según sostienen (Kocalevent et al., 2018). Las culturas individualistas y las colectivistas. Aquellas que apoyan la individualidad y luchan por la independencia de 
los demás, podrían inducir a un menor nivel y estimación del apoyo social. Por el contrario, en las culturas con inclinación hacia lo colectivo, prima el énfasis en la interdependencia armoniosa con otras personas y, en consecuencia, facilita su incremento.

\section{¿Cómo se relacionan el envejecimiento activo y el apoyo social desde una perspectiva psicológica?}

Si bien el apoyo social es una variable en la que no pueden encontrarse grandes diferencias en cuanto a su nivel de percepción según el género, si se pueden encontrar variaciones cuantitativas en base a la edad. Las investigaciones de Kocalevent et al. (2018) demuestran una tendencia general de menor nivel de apoyo social percibido a mayor edad.

Muchos adultos mayores en la actualidad se enfrentan a un grave problema: el aislamiento social y la soledad (Czaja et al., 2018). La falta de recursos financieros suma dificultades a las problemáticas antes mencionadas. Investigaciones realizadas en adultos mayores con bajos ingresos demuestran la importancia de la creación de nuevos contactos y la necesidad de apoyos financieros para desarrollar oportunidades de socialización. Los recursos limitados pueden impedir que participen en actividades sociales. Por lo tanto, la asistencia social preventiva debería dirigir su atención en este sentido.

Un punto para resaltar de las investigaciones realizadas es que no sólo apreciaron el apoyo social recibido. También hicieron hincapié en lo gratificante de brindar apoyo. Tkatch et al. (2017) afirman que existen beneficios en la reciprocidad de los intercambios de apoyo social. Es decir, tanto dar como recibir resultan significativamente beneficiosos. 
Identificar los factores que afectan al apoyo social percibido y documentarlos puede proporcionar información útil y fundada para el desarrollo de programas de intervención efectivos. También pueden optimizarse los recursos disponibles y destinados a determinados sectores de la población. La educación sobre la existencia de grupos de contención disponibles es una posible forma de intervención para disminuir los sentimientos de soledad. Más específicamente para aquellos que son viudos, viven en zonas rurales o tienen restricciones de movilidad. La cuestión es hacer foco en facilitar conexiones significativas entre adultos mayores para mejorar la calidad de vida que llevan y disminuir sus sentimientos de soledad (Kang et al., 2018).

Una herramienta que mostró resultados positivos, según afirman Tkatch et al. (2017), es el uso de líneas telefónicas enfocadas principalmente en crear una red de apoyo para personas que enfrentan problemas o preocupaciones de salud similares.

Las tecnologias de la información y de la computación (T.I.C.) son otra herramienta poderosa con un potencial impacto en la calidad de viday el bienestar (Cotten et al., 2013). Por mencionar un ejemplo, podemos referirnos al proyecto PRISM (Personal Reminder Information and Social Management). Consistió en entregar a los participantes (todos adultos mayores) una computadora con teclado, un mouse, un monitor, acceso gratuito a Internet y una impresora. La computadora entregada llevaba cargado un software llamado PRISM cuyo objetivo era facilitar el acceso a información médica y comunitaria y la autogestión personal (Czaja et al., 2018). 
A partir de los 6 meses de haberse iniciado el proyecto, los participantes reportaron haber recibido mayor apoyo social, a la vez que presentaron una disminución de los sentimientos de soledad y aumentó su sensación de bienestar. Los datos obtenidos en este proyecto respaldan el valor de las tecnologías en general, y más específicamente del software PRISM, como facilitadores de la comunicación. También cabe mencionar, como lo hacen Czaja et al. (2018), que el proceso de capacitación para el uso de estas tecnologías demanda un esfuerzo mental continuoy sostenido. En consecuencia, se pueden experimentar beneficios cognitivos por el entrenamiento. La tecnología, y específicamente el internet, son herramientas viables y útiles para disminuir los niveles de aislamiento social y soledad en adultos mayores.

Algunos de los numerosos beneficios que trae aparejado el apoyo social son: mejor salud, más facilidad para la generación de hábitos, mejor funcionamiento cognitivo y menores niveles de soledad. Estos beneficios no aparecen solamente al recibirlo, sino también al brindarlo (Tkatch et al., 2017).

\section{Conclusiones}

En resumen, el apoyo social es una clave para mantener la salud en la población general, y más específicamente en los adultos mayores. Influye en los niveles de satisfacción con la vida y con los deseos y aspiraciones de las personas. Mientras mayor sea el apoyo que se perciba, menos probable es que se manifieste depresión o sentimientos de soledad. Como contrapartida, su ausencia puede generar sensaciones de soledad con un impacto negativo en la calidad de vida y en la salud. Teniendo en cuenta la propuesta conceptual del envejecimiento activo planteada, podemos 
afirmar con el sustento de diversas investigaciones, que el acompañamiento por parte de redes de apoyo y la sensación real de contar con ellas juega un rol importante en las personas mayores. 


\section{Referencias}

Alsubaie, M. M., Stain, H. J., Webster, L. A. D., \& Wadman, R. (2019). The role of sources of social support on de pression and quality of life for university students. International Journal of Adolescence and Youth, 24(4). https://doi.org/10.1080/02673843.2019.1568887

Cotten, S. R., Anderson, W. A., \& McCullough, B. M. (2013). Impact of Internet Use on Loneliness and Contact with Others Among Older Adults: Cross-Sectional Analysis. Journal of Medical Internet Research, 15(2). https://doi.org/10.2196/jmir.2306

Czaja, S. J., Boot, W. R., Charness, N., Rogers, W. A., \& Sharit, J. (2018). Improving Social Support for Older Adults Through Technology: Findings From the PRISM Randomized Controlled Trial. The Gerontologist, 58(3). https://doi.org/10.1093/geront/gnw249

del Barrio, E., Marsillas, S., Buffel, T., Smetcoren, A.-S., \& Sancho, M. (2018). From Active Aging to Active Citizenship: The Role of (Age) Friendliness. Social Sciences, 7(8).

https://doi.org/10.3390/socsci7080134

García-Torres, M., García-Méndez, M., \& Rivera-Aragón, S. (2017). Apoyo social en adultos mexicanos: validación de una escala. Acta de Investigación Psicológica, 7(1). https://doi.org/10.1016/j.aipprr.2017.02.004

Kang, H.-W., Park, M., \& Wallace (Hernandez), J. P. (2018). The impact of perceived social support, loneliness, and physical activity on quality of life in South Korean older adults. Journal of Sport and Health Science, 7(2). https://doi.org/10.1016/j.jshs.2016.05.003

Kocalevent, R.-D., Berg, L., Beutel, M. E., Hinz, A., Zenger, M., Härter, M., Nater, U., \& Brähler, E. (2018). Social support in the general population: standardization of the Oslo social support scale (OSSS-3). BMC Psychology, 6(1). https://doi.org/10.1186/s40359-018-0249$\underline{9}$

Lin, Y.-Y., \& Huang, C.-S. (2016). Aging in Taiwan: Building a Society for Active Aging and Aging in Place. The Gerontologist, 56(2). https://doi.org/10.1093/geront/gnv107

Marsillas, S., de Donder, L., Kardol, T., van Regenmortel, S., Dury, S., Brosens, D., Smetcoren, A.-S., Braña, T., \& Varela, J. (2017). Does active ageing contribute to life satisfaction for older people? Testing a new model of active ageing. European Journal of Ageing, 14(3). https://doi.org/10.1007/s10433-017-0413-8

Milgrom, J., Hirshler, Y., Reece, J., Holt, C., \& Gemmill, A. W. (2019). Social Support-A Protective Factor for De pressed Perinatal Women? International Journal of Environmental Research and Public Health, 16(8). https://doi.org/10.3390/ijerph 16081426

Rantanen, T., Portegijs, E., Kokko, K., Rantakokko, M., Törmäkangas, T., \& Saajanaho, M. (2019). Developing an Assessment Method of Active Aging: University of Jyvaskyla Active Aging Scale. Journal of Aging and Health, 31(6). https://doi.org/10.1177/0898264317750449

Smith, J. M. (2012). Portraits of Loneliness: Emerging Themes Among Community-Dwelling Older Adults. Journal of Psychosocial Nursing and Mental Health Services, 50(4). https://doi.org/10.3928/02793695-20120306-04

Tkatch, R., Musich, S., MacLeod, S., Kraemer, S., Hawkins, K., Wicker, E. R., \& Armstrong, D. G. (2017). A qualitative study to examine older adults' perceptions of health: Keys to aging successfully. Geriatric Nursing, 38(6). https://doi.org/10.1016/j.gerinurse.2017.02.009

World Health Organisation. (2002). Active ageing: A policy framework (WHO/NMH/NPH/02.8). https:/ / apps.who.int/iris / bitstream / handle / 10665/67215/WH0?sequence $=1$ 